\title{
A Gestão Autônoma da Medicação: uma intervenção analisadora de serviços em saúde mental
}

\author{
Autonomous Medication Management: \\ an analytical intervention in mental health care services
}

\author{
Rosana Teresa Onocko-Campos ${ }^{1}$ \\ Eduardo Passos ${ }^{2}$ \\ Analice de Lima Palombini ${ }^{3}$ \\ Deivisson Vianna Dantas dos Santos ${ }^{1}$ \\ Sabrina Stefanello ${ }^{1}$ \\ Laura Lamas Martins Gonçalves ${ }^{1}$ \\ Paula Milward de Andrade ${ }^{2}$ \\ Luana Ribeiro Borges ${ }^{1}$
}

\footnotetext{
${ }^{1}$ Departamento de Saúde Coletiva, Faculdade de Ciências Médicas,

Universidade Estadual de Campinas. R. Tessália

Vieira de Camargo 126, Unicamp. 13.083-887 Campinas SP. rosanaoc@mpc.com.br ${ }^{2}$ Departamento de Psicologia, Instituto de Filosofia e Ciências Humanas, Universidade Federal Fluminense.

${ }^{3}$ Departamento de

Psicanálise e

Psicopatologia, PósGraduação em Psicologia Social e Institucional, Instituto de Psicologia, Universidade Federal do Rio Grande do Sul.
}

\begin{abstract}
In a context of high rates of medicalization of the population and in light of the scantly critical use of psychiatric medications in mental health services, this paper reports aspects of a qualitative study that had the opportunity to intervene in care practices in three major Brazilian cities. Following the principle of Brazilian Psychiatric Reform championing users' rights to participate in decisions about their treatment, the research intervened in psychosocial care centers (CAPS) seeking the empowerment of the users regarding the use of drugs in their therapeutic projects. Interviews were conducted and focus groups set up. From this recorded material, the paper analyzed some situations that, among other things, attested to the difficulty of avoiding the exercise of power over users via the administration of psychotropic drugs. Little dialogue about drugs, and the existence of stigmatization spaces where user rights are inhibited or "accepted with caution," was also detected in the services surveyed.

Key words Mental health care services, Decision-making, Personal autonomy, Psychotropic drugs
\end{abstract}

Resumo Em um contexto de altas taxas de medicalização da população e face ao uso pouco crítico de medicamentos psiquiátricos em serviços de saúde mental, este artigo reporta aspectos de uma pesquisa qualitativa que teve a oportunidade de intervir em práticas de cuidado em três grandes cidades do Brasil. Seguindo o princípio da Reforma Psiquiátrica brasileira da defesa dos direitos do usuário em participar das decisões sobre seu tratamento, a pesquisa interveio nos centros de atenção psicossocial (CAPS) buscando o "empoderamento" dos usuários em relação ao uso de medicamentos em seus projetos terapêuticos. Foram realizados entrevistas e grupos focais. A partir desse material registrado, o artigo analisou algumas situações que atestaram, entre outras, a dificuldade de evitar o uso do poder sobre os usuários por via da administração de medicamentos psicotrópicos. Também se percebeu, nos serviços pesquisados, pouco diálogo sobre os medicamentos e a existência de espaços de estigmatização onde os direitos dos usuários são inibidos ou aceitos com cautela.

Palavras-chave Serviços de saúde mental, Tomada de decisões, Autonomia pessoal, Psicotrópicos 


\section{Introdução}

No Brasil, até a década de 80 , os hospitais psiquiátricos e os asilos eram os principais locais de tratamento para pessoas com problemas mentais graves. A Reforma Psiquiátrica instituiu uma nova política de saúde mental, que teve, como um de seus principais recursos, o desenvolvimento dos Centros de Atenção Psicossocial (CAPS) para o tratamento em saúde mental na comunidade, possibilitando o seguimento ambulatorial $\mathrm{e}$ a atenção à crise. A transição de um modelo hospitalocêntrico para um de saúde mental comunitária deu-se pela considerável redução dos leitos psiquiátricos e a implantação de serviços substituti$\operatorname{vos}^{1}$. Estes, além das estratégias medicamentosas e psicoterapêuticas, incluem nas suas ações os campos da moradia, do trabalho assistido, do lazer e da cultura como formas legítimas e eficazes na produção de vida e de saúde desta clientela.

Em que pese os inegáveis avanços com novos arranjos em saúde mental, muitos são os desafios a serem enfrentados no que diz respeito a uma efetiva mudança nas práticas de atenção. Um deles diz respeito à primazia do tratamento farmacológico no conjunto de ações dos profissionais de saúde mental, a tal ponto que muitas vezes o tratamento em saúde mental reduz-se apenas aos psicotrópicos ${ }^{2}$.

$\mathrm{Na}$ cultura ocidental, cada vez mais pessoas utilizam intervenções médicas como meio para atingir a transformação de seu próprio eu ${ }^{3}$. A medicalização e a "medicamentalização" da vida vêm crescendo ano a ano ${ }^{4-6}$, imersas em características culturais que fazem com que, no atual modo de vida estadunidense, o que anteriormente seriam características pessoais tornou-se doença ${ }^{7}$. São contextos em que problemas não médicos tornam-se "diagnosticáveis" e "tratáveis" como problemas médicos ${ }^{8}$. À medida que aumenta a série de condições medicalizantes dentro da jurisdição médica, cresce a quantidade de drogas aprovadas para tratá-las?. O número de transtornos mentais reconhecidos cresceu muito nos últimos 60 anos. Mesmo que se considere que a evolução científica está associada a uma maior identificação, melhor especificação e compreensão do que se entende como patológico, não se pode ignorar que é justamente a indústria farmacêutica quem financia grande parte das pesquisas e eventos educativos na área, consolidando-se como uma das indústrias mais lucrativas no mundo e influenciando a produção científica ${ }^{3,7}$.

$\mathrm{Na}$ saúde mental brasileira, estudo anterior apontou que, independente do avanço da Refor- ma Psiquiátrica, a medicalização se mantém como prática não reformada ${ }^{2}$. A hospitalização e a "renovação de receitas" sem a avaliação presencial dos usuários ainda são respostas comuns diante das demandas que aportam ao sistema ${ }^{2}$. Essas práticas, que intencionavam evitar a interrupção dos tratamentos e garantir o cuidado, terminaram por se tornar parte do problema, com indivíduos usando medicamentos por tempo indeterminado e submetidos a um cuidado fragmentado.

Outro aspecto importante é a própria participação do usuário nas decisões relacionadas ao seu tratamento, presente na literatura científica dos últimos 20 anos. Em alguns países, como o Reino Unido, combina-se o que se tem de evidência científica com os valores (incluindo preferências, preocupações, necessidades e desejos) individuais dos usuários e seus familiares ${ }^{10}$. Os guidelines oficiais do sistema nacional inglês deixam explícito que o compartilhamento da decisão com o usuário deve ser condição imperativa para o seguimento de qualquer tratamento ${ }^{11}$. Tratase de algo que se iniciou na saúde mental e tem se expandido para outras áreas da medicina ${ }^{10}$.

No Brasil, a Carta dos Direitos dos Usuários do Sistema Único de Saúde garante premissa similar à do sistema inglês, contudo ainda é pouco praticada no país ${ }^{2}$. A experiência singular e o significado do uso de medicamentos psicotrópicos, para a pessoa envolvida, raramente são levados em conta. A participação dos usuários nas decisões acerca do tratamento restringe-se, muitas vezes, ao mero relato de seus sintomas ${ }^{12-14}$. Portanto, o baixo empowerment poder e autonomia pessoal e coletiva de indivíduos e grupos sociais ${ }^{15}$ que os usuários dos serviços possuem em relação ao seu tratamento, com pouca apropriação de informação e centralização das decisões nos profissionais de saúde, torna a prática clínica mais vulnerável à economia de mercado e ao complexo médico-hospitalar ${ }^{5}$. Daí a importância do protagonismo dos usuários em organizações e em movimentos de empowerment.

Reconhecendo o contexto de utilização pouco crítica dos medicamentos nos tratamentos em saúde mental, bem como o valor simbólico da medicação para aqueles que a utilizam, foi desenvolvida em Quebec (Canadá) uma nova abordagem de intervenção denominada Gestão Autônoma da Medicação (GAM) ${ }^{13,14}$.

A GAM é uma estratégia de alteração das relações de poder para garantir aos usuários efetiva participação nas decisões relativas aos seus tratamentos, o que pressupõe como fundamen- 
tal o diálogo e a troca entre os atores envolvidos no cuidado em saúde mental. Com o suporte de um material impresso (Guia GAM), a proposta é de que os usuários tenham acesso a informações sobre seus tratamentos e assim possam reivindicar seus direitos, dialogando sobre o lugar que a medicação e outras práticas ocupam nas suas vidas, refletindo sobre suas redes sociais e sua qualidade de vida ${ }^{13,14}$.

Nascida no seio dos movimentos sociais de defesa dos direitos dos usuários de saúde mental, a estratégia GAM, no Quebec, teve inicialmente como foco o questionamento da medicação objetivando a sua retirada. Entretanto, o seu desenrolar propiciou a abertura de espaços de diálogo e trocas de experiência de vida e de adoecimento, colocando em cena o reconhecimento de que havia um sofrimento já existente antes do uso de medicamentos, de forma que o foco da estratégia direcionou-se à partilha dos significados desse uso.

Conforme o princípio de que a decisão quanto ao melhor tratamento se faz em uma composição entre os saberes do usuário e da equipe de referência, numa gestão compartilhada do cuidado, e que o exercício de cogestão engendra processos de autonomia ${ }^{16,17}$, realizou-se uma pesquisa-intervenção qualitativa em CAPS de diferentes cidades no Brasil. Fez-se uso, junto a grupos de usuários desses serviços, de uma versão traduzida e adaptada do Guia GAM do Quebec, com vistas à elaboração do Guia GAM brasileiro (Guia GAM-BR) ${ }^{13}$.

A versão brasileira não manteve o tema da retirada da medicação, mas reforçou a tomada de decisões compartilhadas entre usuário e profissional de saúde. O guia é dividido em passos, onde a pessoa é convidada a fazer um balanço da própria vida para alcançar uma melhor qualidade. A dinâmica grupal do uso do Guia visa: a partilha de experiências, a ajuda mútua, o estímulo à busca de informações sobre medicamentos e direitos. As temáticas abordadas incluem questionamentos sobre qualidade de vida, rede social, relacionamentos interpessoais, direitos dos usuários, conhecimento sobre os medicamentos em uso e o papel da medicação na vida de cada um. Ao longo do processo, o guia GAM-BR contribui para a (re)apropriação do poder decisório por parte dos usuários, estimulando que eles participem e discutam com os cuidadores seus tratamentos ${ }^{13}$.

A pesquisa-intervenção não teve, porém, somente um sentido de produção de conhecimento sobre a experiência do uso de psicofármacos nos
CAPS; ela propiciou também uma oportunidade para intervir nas práticas de cuidado em saúde mental $^{18}$. Assumimos a direção indicada pelo movimento institucionalista, na afirmação de que se trata de transformar para conhecer e não de conhecer para transformar a realidade ${ }^{19}$. A análise institucional francesa é uma importante aliada no campo da Reforma Psiquiátrica, na medida em que evidencia a dimensão política da pesquisa quando trata dos processos de institucionalização. Portanto, o objetivo deste artigo é analisar as práticas em saúde mental valendo-se da intervenção da estratégia GAM como deflagradora dessa análise.

\section{Metodologia}

O arranjo operacional utilizado para colocar em prática a estratégia da GAM foi o Grupo de Intervenção (GI). Três GI foram realizados em CAPS, um no Rio de Janeiro (RJ), um em Campinas (SP) e um em Novo Hamburgo (RS); e um quarto GI ocorreu na Universidade Estadual de Campinas (Unicamp-SP). Os GI ocorreram simultaneamente, por dez meses, através de encontros semanais ou quinzenais, perfazendo uma média de vinte encontros em cada GI. Os pesquisadores desempenharam a função de operadores dos grupos, para a qual se fez necessário aliar o conhecimento em pesquisa e a experiência clínica, considerando que o trabalho grupal foi realizado com pessoas que sofrem transtornos mentais graves. Dentre esses pesquisadores, três eram psiquiatras, com experiência de atuação em CAPS. Dois deles atuaram conjuntamente como operadores do grupo de Novo Hamburgo, e o outro em um dos GI de Campinas, atuando em parceria com pesquisadores de outras formações. No GI do Rio de Janeiro, não houve participação de psiquiatras como operadores de grupo.

Os sujeitos que participaram dos grupos de intervenção foram usuários dos serviços de saúde mental e pesquisadores, conforme a Figura 1. Nos grupos, cada participante recebeu um Guia GAM, e este indicava a sequência de temas a serem abordados. Os critérios de inclusão dos usuários que participaram foram: ser portador de transtorno mental grave, usar psicofármacos há mais de um ano, manifestar vontade de participar do grupo. Os critérios de exclusão dos usuários foram: recusa em participar ou em assinar o termo de consentimento livre e esclarecido, limitação cognitiva grave. Para o grupo realizado na Unicamp, foi critério de inclusão também a ca- 


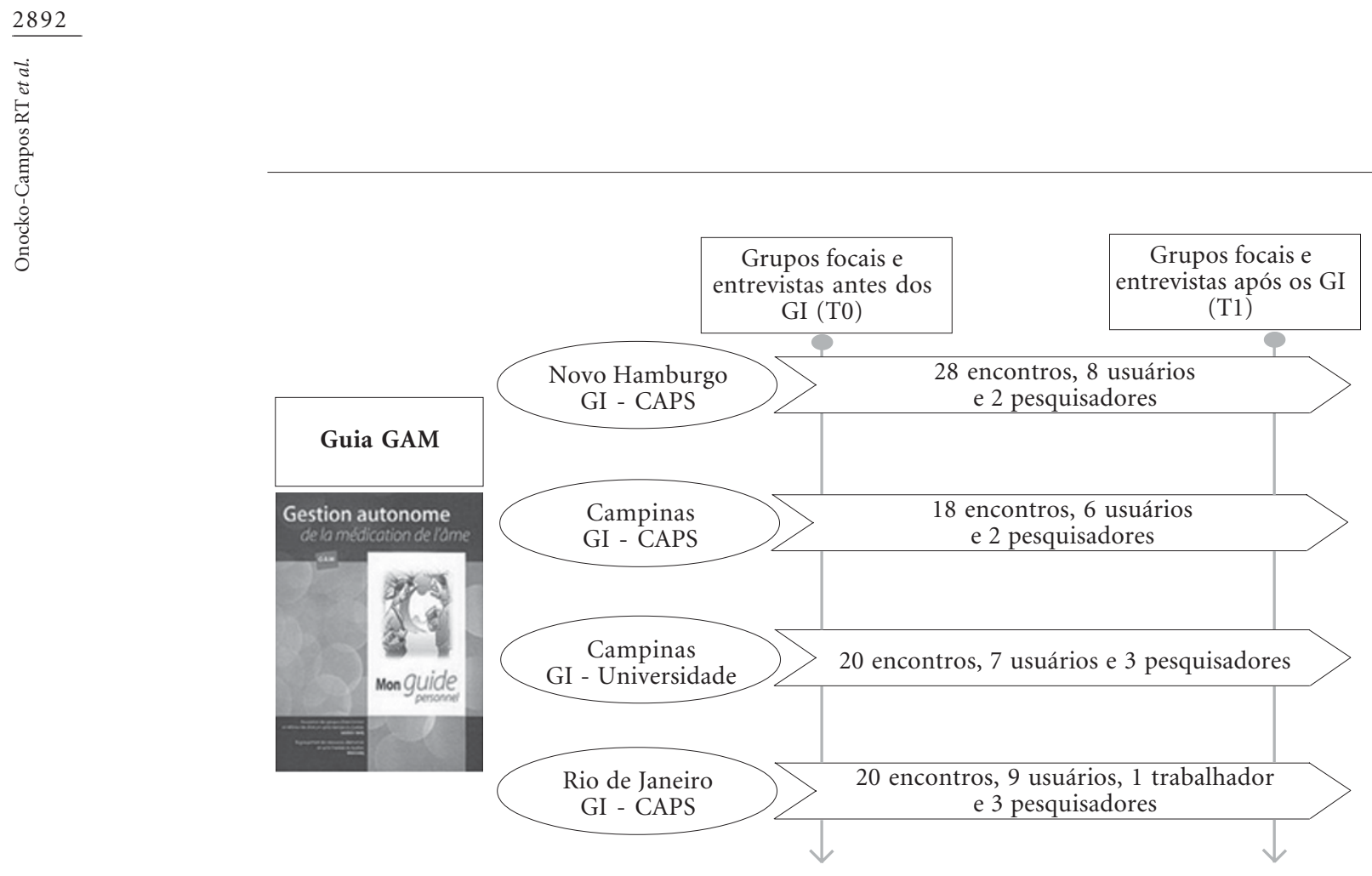

Figura 1. Configuração e participantes dos GI de cada campo

pacidade de circulação pela cidade, pela rede de serviços, e uma trajetória de participação política no campo da saúde mental. O convite aos usuários foi feito nos espaços de reunião e assembleia dos serviços, e a seleção dos participantes, com o intuito de seguir os critérios estabelecidos pela pesquisa, realizou-se de maneira conjunta com os trabalhadores dos serviços onde os grupos aconteceram. Cabe ressalvar que, para alguns dos usuários que participaram dos GI, o tratamento medicamentoso era acompanhado por médicos, não necessariamente do CAPS.

Foram feitos, antes e após o desenvolvimento dos GI, grupos focais com os usuários participantes e entrevistas com gestores e trabalhadores de cada local onde os GI aconteceram. Esses trabalhadores e gestores não necessariamente participaram dos GI - sistematicamente, houve participação de duas trabalhadoras junto aos GI que se realizavam nos CAPS em que atuavam: uma no Rio de Janeiro e outra em Novo Hamburgo. No grupo desenvolvido na Unicamp, pelo fato dos sujeitos terem mais autonomia e transitarem pelos diferentes serviços da rede de saúde, foram realizados grupos focais somente com os usuários. O conteúdo do roteiro, tanto das entrevistas como dos grupos focais, foi: a valorização do contexto do usuário nas condutas clínicas; a capacidade de gestão e compartilhamento de decisões (usuário e equipe); direitos do usuário, em especial no que se refere à medicação (acesso, informação, recusa); a voz do usuário no serviço e na relação médico/paciente; a experiência de uso de psicofármacos; e a visão sobre autonomia de cada um.

Os grupos focais e as entrevistas, realizados por pesquisadores diferentes daqueles que conduziram os GI, foram audiogravados e transcritos integralmente. Essas transcrições deram origem a narrativas que foram construídas por quem conduziu cada grupo ou realizou a entrevista $^{20}$, sendo que cada uma dessas narrativas foi, posteriormente, validada por outro pesquisador. Com os usuários, as narrativas validadas foram a eles apresentadas para outra, final “ $\mathrm{o}$ que temos chamado de grupo focal hermenêuti$\mathrm{co}^{20}$, com base nas formulações acerca da função da narrativa ${ }^{21}$. Os usuários, no encontro com o texto produzido com suas vozes, julgaram se seus pontos de vista estavam ali contemplados, contribuindo para a compreensão dos pesquisadores. $\mathrm{Na}$ análise dessas narrativas, foram extraídos os principais núcleos argumentais que emergiam do material, relacionados ao objetivo da pesquisa.

A pesquisa, com aprovação pelo Comitê de Ética da Universidade Estadual de Campinas, respeitou os aspectos éticos e legais implicados no 
trabalho com pessoas, sobretudo usuárias da rede de saúde mental.

\section{Resultados e Discussão}

Como resultado da intervenção realizada pela pesquisa, surgiram, no contexto dos CAPS, diversas situações analisadoras das suas práticas. Desde a abordagem inicial, gestores e trabalhadores mostraram-se ambivalentes com a estratégia da GAM. Apesar de considerarem importante o empowerment do usuário, num dos campos a equipe pediu para editar partes do Guia GAM antes de usá-lo nos GI, porque temia que a disponibilização de informações sobre os direitos dos usuários e os efeitos colaterais dos medicamentos resultasse em não adesão ou desistência do tratamento.

Nas narrativas dos usuários, os CAPS foram descritos ora como lugar de tratamento e cuidado positivamente avaliado, como fomentador de espaço de escuta, de troca e produção de trabalho; ora como promotor de sentimentos de baixo poder de troca e de participação. Os serviços pareceram estar igualmente marcados por dupla característica: de apoio ao usuário, mas também de tutela.

Em quase todos os grupos focais, os usuários apresentaram dificuldade de dar sentido ao fato de frequentarem o serviço: para mim não está claro por que devo fazer tratamento e vir ao CAPS, ao invés de ficar em casa. (GF usuários 1). Mesmo quando o CAPS fazia sentido para a vida ou era experimentado como um lugar de acolhimento, não promovia, necessariamente, conforme evidenciava a narrativa dos usuários, uma melhor compreensão de sua experiência de adoecimento. Como mencionado em outras pesquisas qualitativas, a relação consigo mesmo, com os outros e com o mundo é abalada pelas situações de crise. E as abordagens psicoeducativas e psicofarmacológicas que predominam nos serviços de saúde mental contribuem para reduzir os espaços para nomear as experiências, elaborá-las e lhes dar um sentido ${ }^{22,23}$.

Nos grupos da GAM, o contato com o médico e com os demais profissionais da equipe de saúde foi estimulado, especialmente no momento em que as dúvidas sobre os medicamentos e os tratamentos surgiram. Em todas as narrativas os usuários relataram que sentiam dificuldade em conversar com o médico e mencionaram a falta de informação que têm sobre os seus próprios tratamentos medicamentosos. Reconhece- ram que falar é muito difícil e apontaram os médicos como detentores da autoridade na relação: Teve um psiquiatra que disse que ia dar os remédios que ele quisesse. Acontece, algumas vezes, de não conseguirmos falar com o médico. A voz não sai, tranca, parece que tem uma coisa que diz assim: - Não fala e não pergunta. (GF usuários 2)

Além disso, mesmo quando o profissional era descrito como mais disponível, sentiam-se, por vezes, vítimas de preconceito: achamos que os médicos dos CAPS têm preconceito com a gente e pensam que não podem deixar a pessoa falar por ser portadora de doença mental. (GF usuários 2).

Alguns usuários relataram que negociariam com seus médicos, não fosse a falta de informação e a dificuldade de comunicação, atreladas à grande demanda e carência de profissionais nos serviços: ... de uma forma geral, achamos que é possível negociar a medicação com os médicos, mas nem sempre podemos. Um de nós, inclusive, nunca perguntou isso, porque achou que eles iriam falar um 'não'. Outro, tem o interesse em fazer isso, mas o único médico [do serviço] é muito ocupado, por isso é difícil conversar. (GF usuários 3).

A mesma questão da insuficiência de pessoal também apareceu nos relatos de gestores e trabalhadores, comprometendo, segundo eles, a qualidade do tratamento oferecido: Ainda existe o fato de a equipe estar sempre reduzida e acaba ficando atrelada ao cuidado dos pacientes intensivos, aqueles mais graves, que permanecem mais tempo aqui, o que impede a saída para fora do CAPS. (Entrevista Gestor 2).

Em que pese tais dificuldades, alguns locais tentavam amenizar os problemas enfrentados pela falta de recursos do serviço: Na tentativa de acompanhar os casos, em uma região que sofre com a pobreza, utiliza-se bastante o transporte disponivel ao CAPS, mas também o próprio carro dos funcionários, pois consideramos o transporte insuficiente e nos vemos sem alternativas. (Entrevista Gestor 3). Referindo-se à grande demanda que chega ao serviço de saúde, um gestor relatou que os atendimentos de grupo são a solução. A utilização de grupos como consultas coletivas, para contornar a falta de recursos, termina por evidenciar uma prática clínica de baixa qualidade, não configurando o que seria esperado de um grupo terapêutico.

Os gestores relataram ainda que os serviços estão mais voltados para tratar das crises já deflagradas do que para dar um seguimento contínuo no tratamento que propiciaria a prevenção delas. Acaba que as parcerias são mais intensas nas situações de crise do que no cotidiano. Quando um 
paciente não está bem, tem que se correr pra montar a rede, o que é um problema, porque acho que idealmente a gente devia estar com essa rede constituída permanentemente. (Entrevista Gestor 1).

Um dos trabalhadores disse sentir-se mais seguro com o trabalho em equipe, mas chamou atenção para a situação atual de falta de pessoal: acabo trabalhando que nem em pronto-atendimento, em que venho aqui, mas não sei mais o que está acontecendo com aquele paciente, não consigo mais fazer seguimento... Então, está bem ruim, sem qualidade nenhuma. Parece que você fica sem retaguarda. O processo de trabalho acaba desestruturando o próprio trabalho... às vezes você é engolido pela demanda. (Entrevista trabalhador 3).

Esses trechos deixam claro o quanto a falta de investimento, a alta rotatividade de pessoal e os recursos insuficientes interferem na qualidade do trabalho prestado. Tal situação já foi evidenciada em pesquisas anteriores, tendo sido destacada como pano de fundo durante nosso estudo ${ }^{24}$.

Os usuários identificaram também que, quando o assunto era medicação, os demais profissionais do CAPS costumavam remeter a decisão ao médico e pareciam pouco apropriados sobre o tema. Este padrão se repetiu em narrativas dos usuários das três cidades, o que mostrou a dificuldade em se discutir o tratamento medicamentoso. Por outro lado, os profissionais médicos relataram que o monopólio das decisões relacionadas aos medicamentos não é algo desejado por eles, mas uma exigência que experimentam em função do modo como se organiza o trabalho no CAPS. Contaram que, no caso de queixas possivelmente associadas a efeitos colaterais, os usuários eram remetidos diretamente a eles, como se outro profissional não pudesse conversar ou abordar essa questão. Reforço a importância do compartilhamento em equipe, ouvir outros pontos de vista, mesmo assim, sinto que a responsabilidade com a medicação está vinculada apenas a mim que sou médico, não sendo tão importante para os outros profissionais. (Entrevista trabalhador 3)

Os trabalhadores não médicos, por sua vez, referiram-se a uma tensão existente entre eles e os médicos, ressaltando que estes têm dificuldade em compartilhar o conhecimento sobre medicamentos, o que, para eles, justificaria o fato dos profissionais não médicos pouco participarem em decisões sobre o assunto. Ao mesmo tempo, identificaram esse monopólio como uma das razões do baixo empowerment dos usuários em relação à sua própria medicação: Entre médico e paciente ainda existe aquela relação de poder; o médico receita o remédio e o paciente deve acei- tar. (Entrevista Trabalhador 1). Por fim, os gestores afirmaram que pouco se debate sobre os medicamentos no cotidiano dos serviços, tanto com os usuários, como entre os profissionais das equipes de saúde mental.

Os usuários ponderaram ainda sobre a timidez, o receio de conversar e "ouvir um não", ou de serem maltratados. Aqueles que expressaram suas ideias para os trabalhadores, após a vivência da estratégia da GAM, indicaram que isso não significou que foram ouvidos: Ganhamos uma lista dos nossos direitos no GAM, mas a gente tem três minutos de consulta pelo SUS, não dá tempo de mostrar. Quando, certa vez, um de nós, tentou mostrar os direitos para o médico, ele desviou ligeiro, deu a receita e já chamou outro. Ele nem leu, nem pegou pra ler, ele viu que a carta era dos direitos, deu a receita e disse pra voltar tal dia. (GF usuários 2). Ainda segundo os usuários, a escuta do médico era seletiva, voltada aos aspectos orgânicos das experiências que lhes eram narradas: Outro colega de nosso grupo conta que os médicos não lhe ouvem. Só ouvem quando tem um problema simples, como: dor de cabeça, cólica... Desses a gente pode falar. (GF usuários 1)

A partir da experimentação dos usuários nos grupos de intervenção da GAM, outra forma de comunicação sobre os psicotrópicos pôde ser vivenciada. Os usuários referiam-se ao grupo como um local de troca de um "saber experiencial" sobre o medicamento, onde cada um pôde contar sua vivência singular com o uso dos psicotrópicos. Ao mesmo tempo, utilizaram essa experiência do GI como um contraponto para criticarem a forma de comunicação dos profissionais que os atendiam. Criticaram a linguagem dos trabalhadores da saúde, definindo-a como técnica e insuficiente para sanar as muitas dúvidas: Como chegaram à conclusão de que alguém é bipolar? Quando vai ser a hora de pararmos o remédio? (GF usuários 2). Sem respostas para suas dúvidas, os usuários revelaram alguns medos, como a impressão de que o tratamento com múltiplos medicamentos pode matar ou causar um estado de coma permanente. Na sua percepção, os médicos orientam pouco sobre o tratamento medicamentoso: falam apenas quanto você vai tomar e em que horário, mas não conversam sobre a adaptação ao remédio (GF usuários 3).

A discussão suscitada pela estratégia da GAM fez com que os usuários chegassem à conclusão de que tratamento e uso regular de medicamento quase se tornaram sinônimos nos CAPS. Eu tenho medo de contar que parei os remédios porque o CAPS é muito apegado a remédio e acho que 
podia ser um pouco mais na conversa também (GF usuários 3). Alguns contaram ainda que já foram ou são ameaçados de serem internados se não tomarem os medicamentos. Outros recursos terapêuticos surgiram nas narrativas, mas não alcançaram o mesmo valor dado ao tratamento psicofarmacológico.

As narrativas desta pesquisa indicam que, nos CAPS onde foi feita a intervenção, não havia suficientes espaços de fala sobre o tratamento medicamentoso com o usuário ou entre os profissionais das próprias equipes. Foram identificados vários problemas associados à dificuldade de comunicação sobre esse tema: relações desiguais de poder, coerção, medo, timidez, uso de linguagem técnica, ausência de escuta para a vivência pessoal e atribuição exclusiva de competências. Considerando-se a mudança do modelo centrado na doença para o modelo de atenção psicossocial preconizada pela política nacional de saúde mental atual, é marcante o fato de que o que mereceu atenção dos trabalhadores, no discurso dos usuários, foi justo aquilo que poderia ser de pronto subsumido ao jargão técnico, de modo que as experiências pessoais não eram ainda consideradas ou ficavam relegadas ao segundo plano. Chamou-nos atenção que, no relacionamento com os trabalhadores dos CAPS, os usuários se sentiram estigmatizados, sendo um dos objetivos desse dispositivo justamente diminuir o preconceito.

A esse respeito, um estudo qualitativo indicou que as intervenções intensivas de saúde mental na comunidade podem ser percebidas como coercitivas e intrusivas pelos usuários, indo contra o princípio de livre escolha e de apropriação do poder, podendo contribuir assim para a estigmatização ${ }^{23}$. Outro estudo, com 120 equipes que realizavam seguimento intensivo, na comunidade, de pessoas com problemas mentais, visando a sua desinstitucionalização, demonstrou que mesmo tal modelo pode veicular a ideia de que a adesão às prescrições farmacológicas é a sua principal finalidade, apesar das evidências científicas dos efeitos limitados desse tipo de tratamento na redução ou eliminação de sintomas face ao processo de restabelecimento e melhora geral na qualidade de vida dos usuários ${ }^{25}$. Mais significativa ainda foi a constatação de que a mudança na política de saúde mental, com a reforma psiquiátrica, impulsionada pelos trabalhadores da saúde ${ }^{1}$, não garantiu mudanças das práticas, predominando o modelo biomédico, centralizando o tratamento no uso de medicamento.

Nos três campos da pesquisa, a forma relatada pelos gestores quanto à atitude do serviço diante de um usuário que, com o juízo crítico preservado, recusa fazer uso de medicamento, varia da não aceitação à aceitação com ressalvas. Alguns trabalhadores relataram que recusar o tratamento sugerido significaria a "não adesão" e, por conseguinte, o desligamento do usuário do serviço. Na recusa do usuário de tomar a medicação, muitos profissionais têm a conduta de fazêlo assinar em seu prontuário e desligá-lo de todo o tratamento. Explica-se que o CAPS está de portas abertas, mas que ele terá que iniciar de novo todo o processo: ir ao posto de saúde, consultar com o médico clínico, pegar o encaminhamento, trazer para cá, fazer o acolhimento, etc. E, caso o usuário faltasse três vezes, por exemplo, ou faltasse duas vezes ao grupo de medicação e uma ao grupo de depressão, seria também desligado do tratamento. Assim, seria desligado aquele que não estivesse cumprindo com o plano terapêutico (Entrevista Gestor 2). Apesar de criticar tal ação, o gestor assume que essa é a prática em seu serviço.

Ainda no discurso de um trabalhador: eu friso que o direito de não usar medicamento não pode ser encarado como se o paciente pudesse fazer tudo o que quer. Eu não costumo dizer aos pacientes diretamente que eles têm o direito de não usar, prefiro salientar que eles devem falar com o médico caso achem que o medicamento está lhes fazendo mal, de modo que possam trocar por outro. (Entrevista trabalhador 1). Em outros relatos, os trabalhadores disseram que não se deve deixar de atender os usuários de forma alguma. No caso destes decidirem não aceitar a medicação ou não frequentar o CAPS, ainda assim, tentariam manter e adaptar o acompanhamento, ora com visitas domiciliares, ora com conversas com os familiares. Entretanto, ao adotarem essa prática, sentiam medo de serem responsabilizados caso algo indesejável acontecesse e fosse associado ao acompanhamento do usuário sem o uso do psicofármaco. Além disso, tem toda uma questão legal que pode me envolver, caso eu banque junto com ele a possibilidade de ficar sem a medicação, em que poderá haver cobranças (Entrevista trabalhador 3 ).

Percebeu-se a existência de um limite tênue entre o cuidado com a saúde dos usuários e o gerenciamento de suas vidas. Considero importante a inclusão do contexto do paciente no atendimento que realizo, pois isso ajuda em muito na administração da vida deles, bem como em meu trabalho...Tentamos fazer o possível para que os pacientes venham ao CAPS diariamente, que tomem os medicamentos aqui. (Entrevista trabalhador 1).

Além disso, quando um usuário piora clinicamente, após a equipe "confiar" na sua capaci- 
dade de seguir corretamente o uso do medicamento prescrito, há dificuldade em reinvestir na corresponsabilização e permitir que o usuário autoadministre sua medicação, por considerarem que, quando ele se sentir bem, interromperá novamente seu uso. Com o grupo de intervenção da GAM, alguns trabalhadores perceberam que os usuários reclamaram de não poder discutir a medicação com o psiquiatra e os demais profissionais da equipe. Identificaram que exatamente os usuários mais críticos eram os que menos aderiam aos dispositivos clínicos ofertados pelo CAPS e não seguiam "corretamente" o tratamento indicado. Esses trabalhadores não se indagaram se os tratamentos oferecidos no CAPS atendiam aos desejos dos usuários, que fizeram reclamações apoiados em seus próprios objetivos e expectativas com o tratamento.

Verificamos, a partir do que se mostrou na pesquisa, que o usuário frequentemente ocupa uma das duas posições frente ao tratamento prescrito: a de sujeito ou com pouca voz e pouca participação no projeto terapêutico atribuído ou excluído de seu próprio direito ao tratamento, correndo o risco de tampouco ter apoio da família que, muitas vezes, reforça e garante sua adesão irrestrita ao prescrito no serviço de saúde. Nos casos de exceção, em que os usuários mostraram maior protagonismo em seu tratamento, os trabalhadores pareciam manter uma ligação mais frágil com eles e, ao mesmo tempo, enfrentavam a oposição de colegas, o medo de responsabilização e punição pelo que pudesse advir a esses usuários em função de seu protagonismo.

Nas entrevistas com os gestores, foram referidas diversas situações em que se justificava a impossibilidade do manejo de crise sem o uso de psicotrópicos: É o nosso mandato, se a gente não fizer, o paciente estará aí, correndo risco... fazendo alguém correr risco. Não é desejável que ele vá parar no campo jurídico ou penal. Às vezes, tem risco que você não imagina! (Entrevista Gestor 1). Essas falas evidenciaram a predominância do papel de tutela sobre o de cuidado.

Nos momentos descritos como aqueles em que o usuário se coloca em risco ou expõe outros a risco, foi defendido um agir de forma mais "firme", impondo a internação e/ou o uso do medicamento, mesmo que involuntariamente. Justificou-se como um cuidado necessário, ao julgarem extintas as possibilidades de negociação. Quando o usuário se coloca numa situação de ris$c o$, a gente obrigatoriamente interfere (Entrevista Gestor 1). Neste ponto, todos os discursos foram congruentes, apontando para ações invo- luntárias como forma de proteção. Infelizmente, não foram exploradas, no estudo, quais situações eram consideradas de risco pelas equipes e qual a possibilidade de negociação. Não houve menção à possibilidade da elaboração de um plano para a crise, combinado previamente com o usuário, corroborando a ideia de que há dificuldade de inclusão do usuário na negociação da direção do seu tratamento.

No momento em que o Guia GAM abordou os direitos dos usuários, estes apontaram os espaços onde está previsto que falem: as "assembleias", a associação de usuários do CAPS e outros grupos. Nos espaços criados para garantir a democracia institucional, a participação é acolhida e respeitada, segundo os próprios usuários. Todavia, quando o assunto está relacionado à gestão de seu próprio tratamento, como o direito de recusá-lo, os usuários não se sentiram ouvidos: se isso ocorre [recusa ao medicamento], põem remédio pela garganta, dão injeção ou, na internação, amarram logo (GF usuários 1). A recusa ao tratamento medicamentoso coloca o usuário diante da ameaça de ser transferido para um lugar pior ou de desencadear uma nova crise, causando o retorno ao uso dos psicotrópicos.

Após as discussões abordando os direitos dos usuários nos GI, alguns fizeram pedidos às equipes dos CAPS para ter acesso ao prontuário e à bula de seus medicamentos, porém sentiram dificuldades em obter o que reivindicavam. Questionaram também a impossibilidade de escolha do profissional que os atendia e os empecilhos para conversar com as pessoas com quem se sentiam mais à vontade. Eu liguei para o CAPS e falei que queria parar os remédios, que queria conversar com a médica de quem eu mais gosto. E eles falaram que quem vai me atender é uma outra que eu não gosto. O paciente não pode escolher quem ele quer que seja sua referência (GF usuários 3). Estudo brasileiro já apontou essa mesma dificuldade na atenção básica ${ }^{26}$.

A partir dos objetivos desta pesquisa-intervenção, cujas práticas-alvo foram o compartilhamento de decisões e a garantia do direito dos usuários a participarem de seus próprios tratamentos, a metodologia de trabalho da GAM foi vista como algo não habitual, seja para os usuários, seja para os trabalhadores e os gestores. Em que pese os seus méritos, a Reforma Psiquiátrica brasileira foi fundamentalmente uma reforma estrutural, com aumento do financiamento aos serviços extra-hospitalares de base comunitária, resultando na forte expansão do número de CAPS em território nacional ${ }^{27} \mathrm{e}$, mais recentemente, na 
implementação de redes de atenção psicossocial. Entretanto, no tangente aos tratamentos medicamentosos, na forma como são oferecidos, a prática ainda não superou o modelo biomédico prevalente. Além disso, subsistem, nesses serviços, espaços de controle, de dominação e estigmatização, onde os direitos dos usuários são inibidos ou aceitos com cautela.

Alguns autores, como Bleger ${ }^{28}$, já identificaram a tendência das organizações em reproduzir os problemas que elas se propõem responder. Em outro estudo qualitativo, alguns CAPS estudados se apresentaram como espaços de captura, de anulação da potência dos coletivos de usuários ${ }^{29}$. A tendência à tutela, existente em diversos serviços de saúde, justificada pelos "riscos potenciais" de um usuário de serviços de saúde mental para a sociedade, faz persistir a ideia de que o usuário não é capaz de discernir o que é o melhor para ele, reforçando sua exclusão no processo decisório de seu tratamento ${ }^{30}$.

O tratamento medicamentoso revelou-se um assunto pouco debatido, seja pelos usuários, seja pelos trabalhadores. Percebeu-se que os trabalhadores não médicos pouco se apropriaram do tema, endereçando as decisões acerca da prescrição medicamentosa exclusivamente aos médicos. Sem deter esse saber, como poderão incentivar os usuários a dialogar e a negociar com os seus prescritores, a fim de incluir o saber experiencial no projeto terapêutico? Esta questão torna-se mais premente em um contexto onde o conceito de tratamento raramente se divorcia da prescrição medicamentosa.

A pesquisa levou-nos à constatação de que ainda são necessárias mudanças nas práticas em saúde mental, especialmente no que se refere à valorização da experiência do usuário em seu tratamento. Estimular a autonomia e o poder de agir dos usuários e mobilizá-los para que descubram ou redescubram seus interesses e desejos, segue sendo um desafio para o cuidado em saúde mental - um cuidado cujas principais preocupações deixem de ser o diagnóstico, a doença e a prescrição medicamentosa. A direção proposta é que o usuário, em vez de ocupar um lugar de dependência na relação com o serviço, tenha o serviço como espaço a partir do qual retome o seu lugar de cidadão. Tem-se como exemplo, a partir do relato de usuários, o Movimento de Saúde Mental Comunitária do Bom Jardim em Fortaleza $(\mathrm{CE})^{31}$. Através de iniciativas como a estratégia da GAM, afirmamos a possibilidade de avançar, para além de uma reflexão sobre as práticas, em direção a mudanças e à possibilidade de um cuidado compartilhado em saúde.

\section{Colaboradores}

RT Onocko-Campos, E Passos, AL Palombini, DVD Santos, S Stefanello, LLM Gonçalves, PM Andrade e LR Borges trabalharam juntos em todas as etapas do manuscrito. 


\section{Referências}

1. Jacob KS, Sharan P, Mirza J, Garrido-Cumbrera M, Seedat S, Mari JJ, Sreenivas V, Saxena S. Global mental health 4 - mental health systems in countries: Where are we now? Lancet 2007; 370(9592):1061-1077.

2. Onocko Campos R, Gama CA, Ferrer AL, dos Santos DVD, Stefanello S, Trape TL, Porto K. Mental health in primary care: An evaluative study in a large brazilian city. Cien Saude Colet 2011; 16(12): 4643-4652.

3. Elliot C. Better then well: American medicine meets the american dream. New York: W.W. Norton; 2003.

4. Bell SE, Figert AE. Medicalization and pharmaceuticalization at the intersections: Looking backward, sideways and forward. Soc Sci Med 2012; 75(5):775783.

5. Conrad P, Leiter V. Medicalization, markets and consumers. J Health Soc Behav 2004; 45(Supl.):158 176.

6. Illich I. A expropriação da saúde: Nímesis da mecicina. 2a Edição. Rio de Janeiro: Nova Fronteira; 1975.

7. Elliot C. White coat, black hat: Adventures on the dark side of medicine. Boston: Beacon Press; 2010.

8. Conrad P, Barker KK. The social construction of illness: Key insights and policy implications. $\mathrm{J} \mathrm{He}$ alth Soc Behav 2010; 51(Supl.):S67-79.

9. Conrad P, Mackie T, Mehrotra A. Estimating the costs of medicalization. Soc Sci Med 2010; 70(12):1943-1947.

10. Fulford KWM. Bringing together values-based and evidence-based medicine: UK department of health initiatives in the 'personalization' of care. J Eval Clin Pract 2011; 17(2):341-343.

11. Deegan PE, Drake RE. Shared decision making and medication management in the recovery process. Psychiatr Serv 2006; 57(11):1636-1639.

12. Lopes TS, Dahl CM, Serpa Júnior OD, Leal EM, Campos RTO, Diaz AG. The process of recovery in the perspective of persons with schizophrenia spectrum disorders and of psychiatrists working at psychosocial health care services. Saúde Soc. São Paulo 2012; 21(3):558-571.

13. Onocko Campos RT, Palombini AL, Silva AE, Passos E, Leal EM, Serpa Júnior OD, Marques CC, Gonçalves LLM, Santos DVD, Surjus LTLS, Arantes RL, Emerich BF, Otanari TMC, Stefanello S. Multicenter adaptation of the guide for autonomous management of medication. Interface (Botucatu) 2012; 16(43):967-980.

14. Regroupement des ressources alternatives en santé mentale du Québec (RRASMQ). Balises pour une approche alternative des pratiques de soutien communautaire en santé mentale. Québec: RRASMQ; 2006.

15. Vasconcelos EM. Dispositivos associativos de luta e empoderamento de usuários, familiares e trabalhadores em saúde mental no brasil. Revista Vivência 2007; (32):173-206.

16. Campos GWS. Um método para análise e co-gestão de coletivos. São Paulo: Hucitec; 2000.

17. Onocko Campos RT, Campos GWS. Co-construção de autonomia: $\mathrm{O}$ sujeito em questão. In: Minayo MCS, Akerman M, Drumond Júnior M, Carvalho YM, organizadores. Tratado de Saúde Coletiva. São Paulo: Hucitec; 2006. p. 669-714.
18. Passos E, Barros RB. A cartografia como método da pesquisa-intervenção. In: Kastrup V, Escóssia L, organizadores. Pistas do método da cartografia: pesquisa-intervenção e produção de subjetividade. Porto Alegre: Sulina; 2009. p. 17-31.

19. Lourau R. Objeto e método da análise institucional. In: Altoé S, René Lourau R, organizadores. Analista em tempo integral. Campinas: Hucitec; 2004. p. 66-86.

20. Onocko Campos RT, Furtado JP. Narratives: Use in qualitative health-related research. Rev Saude Publica 2008; 42(6):1090-1096.

21. Ricouer P. Tempo e narrativa. Campinas: Papirus; 1997.

22. Corin E. Se rétablir après une crise psychotique: Ouvrir une voie? Retrouver sa voix? Santé Mentale Au Quebéc 2002; 27(1):65-82.

23. Rodriguez L. Nouveaux paramètres pour l'élaboration des pratiques de soutien communautaire: Contribuition des organismes communautaires et alternatifs au québec. Santé Mentale Au Quebéc 2011; 36(1):35-56

24. Santos NR. [Public healthcare policy in brazil: Crossroads and choices]. Cien Saude Colet 2008; 13(Supl. 2):2009-2018

25. Davidson L, Drake RE, Schmutte T, Dinzeo T, Andres-Hyman R. Oil and water or oil and vinegar? Evidence-based medicine meets recovery. Community Ment Health J 2009; 45(5):323-332.

26. Onocko Campos RT, Campos GW, Ferrer AL, Correa CR, Madureira PR, Gama CA, Dantas DV, Nascimento R. Evaluation of innovative strategies in the organization of primary health care. Rev Saude Publica 2012; 46(1):43-50.

27. Goncalves RW, Vieira FS, Delgado PG. Mental health policy in brazil: Federal expenditure evolution between 2001 and 2009. Rev Saude Publica 2012; 46(1):51-58

28. Bleger J. Psico-higiene e psicologia institucional. Porto Alegre: ArtMed Editora; 1984.

29. Figueiró RA, Dimenstein M. The daily life of users of CAPS: Empowerment or capture? Fractal: Rev. de Psicologia 2010; 22(2):431-446.

30. Carson D. Good enough risk taking. Int Rev Psychiatry 1997; 9(2-3):303-308.

31. Silva Melo AK, Godoy MGC, Bosi MLM, Ximenes VM, Carvalho LB. Inovação em saúde mental sob a ótica de usuários de um movimento comunitário no nordeste do brasil. Cien Saude Colet 2012; 17(3):643-651.

Artigo apresentado em 25/03/2013

Aprovado em 28/04/2013

Versão final apresentada em 11/05/2013 
Ciência \& Saúde Coletiva

volume 18 número 10 - 2013

p. 2889

onde se lê:

Laura Lamas Magalhães Gonçalves

leia-se:

Laura Lamas Martins Gonçalves

volume 19 número 2 - 2014

p. 609

onde se lê:

Pharmaceutical care and evenly hovering attention:

commitment formations between pharmacy and psychoanalysis

leia-se:

Pharmaceutical care and suspended attention:

compromise-formations between pharmacy and psychoanalysis 